\title{
What are the mechanisms underlying vaping-induced lung injury?
}

\author{
Laura E. Crotty Alexander,, ${ }^{1,2}$ Amy L. Bellinghausen, ${ }^{2}$ and Michelle N. Eakin ${ }^{3}$ \\ 'Pulmonary and Critical Care Section, VA San Diego Healthcare System, La Jolla, California, USA. ²Division of Pulmonary, Critical Care and Sleep Medicine, Department of Medicine, UCSD, La Jolla, California, \\ USA. ${ }^{3}$ Division of Pulmonary and Critical Care, Department of Medicine, Johns Hopkins University, Baltimore, Maryland, USA.
}

E ectronic cigarette (e-cigarette) use and vaping have dramatically increased in recent years, particularly among teenagers and young adults, with an estimated $7.6 \%$ reporting current, everyday e-cigarette use and $27.5 \%$ reporting use in the past 30 days $(1,2)$. The exact numbers of nicotine vapers who also vape marijuana are unknown, but there is significant overlap, with young adults who use e-cigarettes having an adjusted odds ratio of concurrent marijuana use of 3.47 (3).

In 2019, e-cigarette or vaping product use-associated lung injury (EVALI) was recognized as a unique disease entity, and rapid escalation of the numbers of those affected was such that it has been labeled a public health crisis, with over 2800 cases of hospitalizations and 68 deaths as of March 2020. Individuals affected are predominantly males (66\%) who use tetrahydrocannabinol-containing (THC-containing) vapes (82\%). However, many EVALI patients report using both nicotine and THC products (43\%) (4). Interestingly, there is significant clinical overlap between the EVALI epidemic and the COVID-19 pandemic. Both of these diseases have substantial gastrointestinal symptoms, bilateral lung infiltrates, and significant numbers of those affected progressing to hypoxic respiratory failure and acute respiratory distress syndrome (ARDS). But whereas EVALI is mainly a disease of the young, with a median age of 24 years, severe COVID-19 is primarily a disease of the elderly. Although smoking has been linked to increased COVID-19 severity (5), it is as yet unknown whether vaping increases the susceptibility to or severity of this novel viral illness.

At the core of EVALI and COVID-19 is the clinical syndrome of acute lung injury (ALI), which is defined by injury to both alveolar epithelial cells and pulmonary vascular endothelial cells throughout both lungs. The damage to epithelial and endothelial cells leads to destruction of alveolar-capillary membrane integrity, with fluid filling the alveoli, migration of neutrophils into the lungs, and release of both proinflammatory and cytotoxic factors (6). The exact mechanism behind the lung injuries seen in EVALI is unknown, but we propose two possibilities (Figure 1A): (a) a chemical inhaled within the aerosol created by vaping or dabbing is directly cytotoxic to certain lung cells, leading to cellular necrosis, neutrophilic inflammation, and collateral damage, or, alternatively, (b) a two-hit phenomenon occurs, in which inhalation of the base ingredients of e-liquids (propylene glycol and glycerin) within e-device-derived aerosols leads to alterations in the homeostatic state of lung immune cells, such that when a normally well-tolerated inhalant hits the lungs, it tips the balance and triggers massive inflammation. This JCI Viewpoint article discusses these potential mechanistic pathways in the context of how other lung inhalation injuries occur and pinpoints important research needs moving forward.

\section{Epidemiology of vaping and the EVALI epidemic}

As the popularity of vaping has risen, there has been a profusion of novel flavor additives, particularly fruit and candy flavors. Nontobacco flavors appeal more to younger e-cigarette users (7) and are associated with a greater likelihood of initiation and continued use over time $(8,9)$. Many of the chemicals used to create particular flavors contain known toxins, including diacetyl and 2,3-pentanedione (10). Complex

Conflict of interest: The authors have declared that no conflict of interest exists.

Copyright: ( 2020, American Society for Clinical Investigation.

Reference information: J Clin Invest. 2020;130(6):2754-2756. https://doi.org/10.1172/JCI138644.

chemical reactions occur during heating of the e-liquid, generating additional bioactive compounds that may be damaging to the lungs.

In addition to flavored nicotine solutions, cannabis products are frequently used in e-devices. Up to $61 \%$ of cannabis users report vaping a THC product at some time in their life (11). This includes vaping THC liquids, vaping cannabidiol (CBD) oils added to regular e-liquids, and dabbing highly concentrated THC substances. Unfortunately, exact statistics on cannabinoid vaping and dabbing are not available, making accurate assessments of their incidence impossible.

\section{Mechanisms of direct lung injury by inhalants}

To support our direct toxicity hypothesis (Figure 1), there are multiple chemicals known to cause inflammation when inhaled. Diacetyl, a chemical added to e-liquids to give a buttery flavor, is known to cause bronchiolitis obliterans (popcorn lung), an obstructive disease of the small airways (12). The mechanism of this disease is not fully understood but is believed to be due to direct damage to the respiratory epithelium, with disorganized fibrotic repair (13). Nicotine itself is believed to be the causal agent in acute eosinophilic pneumonia, seen in both cigarette smokers and e-cigarette vapers, but the mechanism remains unknown $(14,15)$. In EVALI, the neutrophil, not the eosinophil, is the predominant inflammatory cell recruited to the lungs, which makes the disease more pathologically similar to ALI (16). EVALI is also similar to acute interstitial pneumonia (AIP), in that neutrophils are the main cells driving inflammation and AIP rapidly responds to steroid treatment.

Chlorine is another well-known chemical cause of ALI and ARDS (17), and its pattern of lung damage may best parallel that seen in EVALI. In chlorine pulmo- 
A

Hypothesis 1 Direct chemical injury

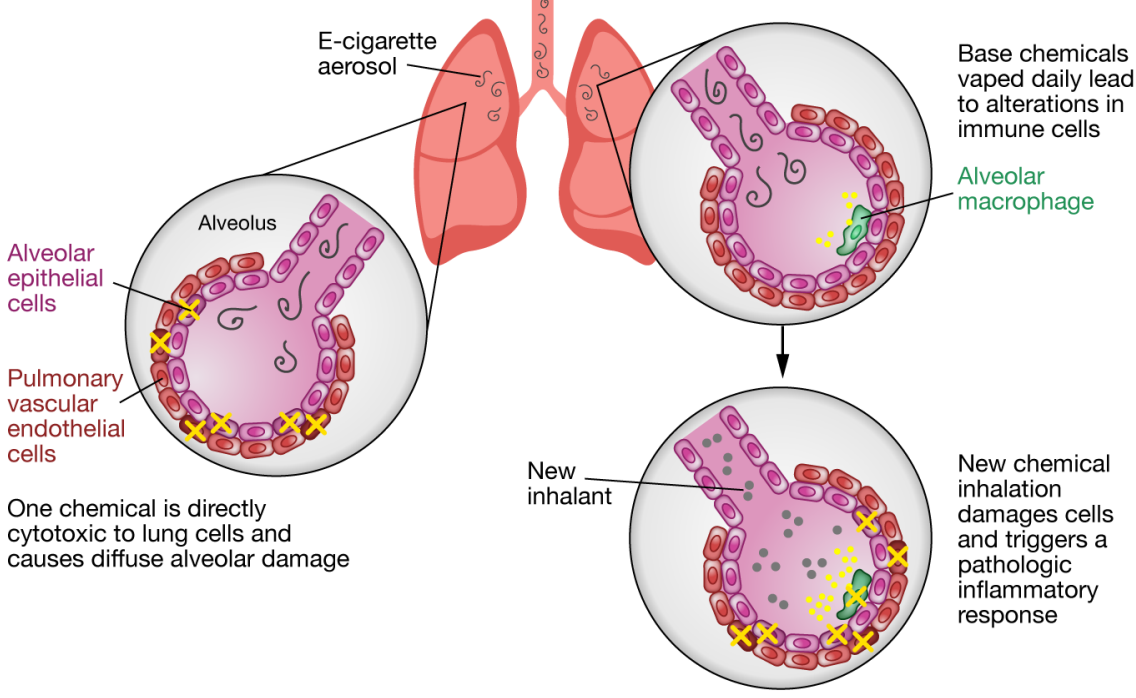

B

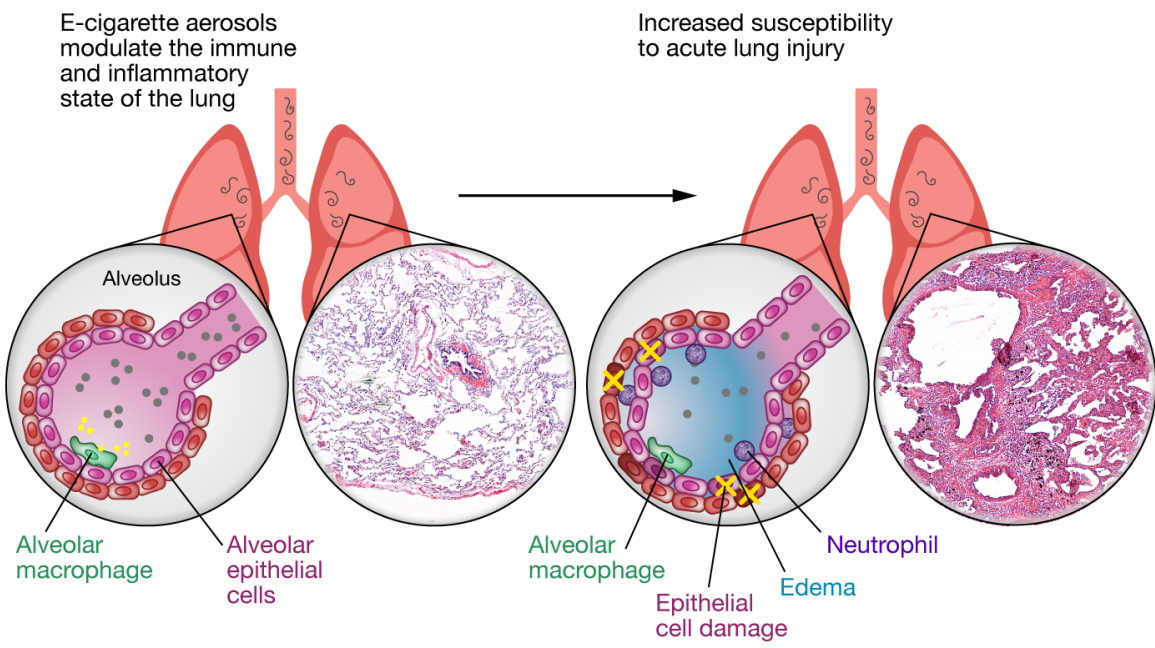

Figure 1. Mechanisms underlying lung injury in EVALI. (A) In the panel on the left, cells of the lung are damaged by one chemical inhaled within e-cigarette aerosols. This cell damage leads to epithelial and endothelial cell necrosis (indicated by a yellow " $X$ "). An alternative hypothesis is depicted on the right, in which inhalation of chemicals common in e-cigarette aerosols (propylene glycol, glycerin, nicotine, or THC) alters the inflammatory state of the lungs, including changing alveolar macrophages to a proinflammatory phenotype with the release of inflammatory cytokines (indicated by yellow circles), such that a second hit causes a pathologic inflammatory response. (B) The immune state of the lungs is known to be altered by the chronic, daily inhalation of e-cigarette aerosols. It is likely that vitamin $E$ is directly cytotoxic to lung cells (indicated by a yellow " $X$ "), leading to necrosis, neutrophil recruitment and activation resulting in collateral damage, and noncardiac pulmonary edema. Illustration credit: Rachel Davidowitz. H\&E-stained lung tissues are from the UCSD Liebow Collection, 1974.

nary toxicity, chloride gas $\left(\mathrm{Cl}_{2}\right)$ combines with water in the airway mucosa to form hydrochloric acid $(\mathrm{HCl})$ and hypochlorous acid (HOCl), both of which directly damage the airway epithelial surface. Reactive nitrogen compounds are also formed by increased activity in the inducible nitric oxide synthase (iNOS) pathway. Furthermore, neutrophil recruitment and mito- chondrial dysfunction lead to additional oxygen radical formation. This results in diffuse lung damage and ARDS, similar to what is observed in EVALI. Phosgene gas (carbonyl dichloride), used as a bioweapon during World War I, similarly causes ARDS by direct cellular toxicity and increased free radical generation (18). The decreased water solubility of phosgene (compared to chlorine) results in a delay of symptom onset, which may be a factor in the inconsistent time course of EVALI.

\section{Vitamin E as a potential culprit}

Currently, the prime suspect molecule in EVALI is vitamin $\mathrm{E}$, which has been identified in many e-liquids and bronchoalveolar lavage (BAL) samples from affected individuals (19). Little is known about the nondermatologic and nongastrointestinal ingestion effects of vitamin $\mathrm{E}$. It is possible that the inhalation of this large molecule leads to direct cytotoxicity of particular lung cell types, versus an attempted clearance of this molecule by alveolar macrophages that leads to accumulation within the vacuoles due to an inability of these cells to break it down, followed by macrophage cell death or promotion of a proinflammatory macrophage phenotype that drives ALI (Figure 1B).

\section{Proposed interventions and public policy}

In order to prevent the considerable harms linked to EVALI and ongoing e-cigarette use, it is important to consider public health policies to both prevent the use of e-cigarettes by young people and identify evidence-based interventions to help e-cigarette users quit. Given the heterogenous potential mechanisms for EVALI, any intervention to decrease vaping-associated lung injury will need to be sufficiently flexible to cover the spectrum of possible causative agents. Although legislation to ban flavor additives in prefilled cartridges except for tobacco and menthol has been passed, this does not address either nonflavor-related lung toxicity or the flavored e-liquids used in refillable or disposable vaping devices. Given that EVALI has been observed in individuals who do not use flavors other than tobacco or menthol, it is likely that this intervention will not eliminate EVALI. Additionally, the current podbased partial flavor ban policy is unsatisfactory, since it leaves menthol products and other flavorings available for other vaping products that have been shown to appeal to youth.

If a flavor ban is not the solution to EVALI, what is the alternative? We propose that increased labeling of e-liquid contents based on mass spectrographic analysis is an important first step. Improved 
characterization of chemical components in e-liquid will allow researchers to better identify possible pulmonary toxins in future cases of EVALI. Furthermore, it will empower consumers of e-liquids to limit their exposure to potentially harmful additives by increasing awareness of what they are inhaling. A drawback to this approach is that it is time consuming and costly, meaning that legislation would likely be necessary to motivate manufacturers of e-liquid to increase labeling.

Tobacco control policies, such as raising the minimum age to purchase tobacco products to 21 , which was recently passed in the US, are critical to reduce youth access. Another target for public policy intervention would be to limit the nicotine content of e-liquid, with the aim of reducing the addictiveness of vaping. It has been hypothesized that the limit of nicotine to less than $20 \mathrm{mg} / \mathrm{mL}$ in the United Kingdom is the main reason that vaping is not as prominent in their youth. Additionally, legislation prohibiting e-cigarette marketing to adolescents and young adults should be strengthened to prevent future generations of youth from acquiring the habit. If vaping and e-cigarette use can be reduced, not only will we reduce the incidence of EVALI, but we will hopefully prevent the (as-yet unknown) long-term health consequences of vaping.

There are currently no evidencebased interventions to help individuals stop using e-cigarettes. Interventions that are currently being promoted or recommended have been modified from existing counseling interventions for helping individuals quit combustible tobacco use and have not been directly evaluated in e-cigarette users or validated in children or teenagers, a population that is now in need of treatment for nicotine addiction. There is a critical need for increased funding for research to identify evidence-based interventions that are tailored for e-cigarette cessation across age groups.

\section{Where do we go from here?}

The potential mechanisms for EVALI are diverse (Figure 1). Investigators have identified vitamin $\mathrm{E}$ acetate as a possible causative agent of EVALI, however, it is likely that additional damaging compounds will be identified as the research continues. Exploration of other toxic inhalation syndromes as well as utilization of animal models to assess the cytotoxic and inflammatory effects of various chemicals in THC vaping and dabbing devices may serve to better elucidate the mechanisms of lung injury in vaping-associated lung disease.

There is a critical need for a broad research agenda to address the public health epidemic of EVALI (20). The CDC and FDA have been working with researchers to track cases and identify possible mechanisms or exposures linked to the development of this disorder. Further research is needed to more directly clarify the precise mechanism of action to both treat and prevent EVALI as well as vaping-induced lung diseases in general. In particular, it is critical that biomedical researchers be allowed to study all THC and nicotine products being used by the general public. Finally, there is a need for broad tobacco control policies to prevent access to these products and identify evidence-based interventions to support cessation.

\section{Acknowledgments}

LECA's salary was supported in part by the VA San Diego Healthcare System and by grants from the NIH (R01HL147326, to LECA) and the Tobacco-Related Disease Research Program (TRDRP T30IP0965 and 26IP-0040, to LECA). ALB is supported by a training grant from the National Heart, Lung, and Blood Institute of the NIH (T32HL134632).

Corresponding Author: Laura E. Crotty Alexander; 9500 Gilman Drive, MC 9111J, San Diego, California 92093, USA. Phone: 619.438.4207; Email:1ca@ucsd.edu.

1. Dai H, Leventhal AM. Prevalence of e-cigarette use among adults in the United States, 20142018. JAMA. 2019;322(18):1824-1827.

2. Cullen KA, et al. e-Cigarette use among youth in the United States, 2019. JAMA. 2019;322(21):2095-2103.

3. Chadi N, Schroeder R, Jensen JW, Levy S. Association between electronic cigarette use and marijuana use among adolescents and young adults: a systematic review and meta-analysis [published online August 12, 2019]. JAMA Pediatr. https://doi.org/10.1001/jamapediatrics.2019.2574

4. Perrine CG, et al. Characteristics of a Mul- tistate Outbreak of Lung Injury Associated with E-cigarette Use, or Vaping - United States, 2019. MMWR Morb Mortal Wkly Rep. 2019;68(39):860-864.

5. Berlin I, Thomas D, Le Faou AL, Cornuz J. COVID-19 and smoking [published online April 3, 2020]. Nicotine Tob Res. https://doi. org/10.1093/ntr/ntaa059.

6. Johnson ER, Matthay MA. Acute lung injury: epidemiology, pathogenesis, and treatment. JAerosol Med Pulm Drug Deliv. 2010;23(4):243-252.

7. Harrell MB, et al. Flavored e-cigarette use: characterizing youth, young adult, and adult users. Prev Med Rep. 2017;5:33-40.

8. Leventhal AM, et al. Flavored E-cigarette use and progression of vaping in adolescents. Pediatrics. 2019;144(5):e20190789.

9. Villanti AC, et al. Association of flavored tobacco use with tobacco initiation and subsequent use among US youth and adults, 2013-2015. JAMA Netw Open. 2019;2(10):e1913804.

10. Allen JG, et al. Flavoring chemicals in E-cigarettes: diacetyl, 2,3-pentanedione, and acetoin in a sample of 51 products, including fruit-, candy-, and cocktail-flavored E-cigarettes. Environ Health Perspect. 2016;124(6):733-739.

11. Lee DC, Crosier BS, Borodovsky JT, Sargent JD, Budney AJ. Online survey characterizing vaporizer use among cannabis users. Drug Alcohol Depend. 2016;159:227-233.

12. Kanwal R. Bronchiolitis obliterans in workers exposed to flavoring chemicals. Curr Opin Pulm Med. 2008;14(2):141-146.

13. Harber P, Saechao K, Boomus C. Diacetylinduced lung disease. Toxicol Rev. 2006;25(4):261-272.

14. Crotty Alexander LE, Shin S, Hwang JH. Inflammatory diseases of the lung induced by conventional cigarette smoke: a review. Chest 2015;148(5):1307-1322.

15. Arter ZL, Wiggins A, Hudspath C, Kisling A, Hostler DC, Hostler JM. Acute eosinophilic pneumonia following electronic cigarette use. Respir Med Case Rep. 2019;27:100825.

16. Layden JE, et al. Pulmonary illness related to E-Cigarette use in Illinois and Wisconsin - Final Report. NEngl JMed. 2020;382(10):903-916.

17. White CW, Martin JG. Chlorine gas inhalation: human clinical evidence of toxicity and experience in animal models. Proc Am Thorac Soc. 2010;7(4):257-263.

18. Li W, Pauluhn J. Phosgene-induced acute lung injury (ALI): differences from chlorine-induced ALI and attempts to translate toxicology to clinical medicine. Clin Transl Med. 2017;6(1):19.

19. Blount BC, et al. Evaluation of bronchoalveolar lavage fluid from patients in an outbreak of E-cigarette, or vaping, product use-associated lung injury - 10 states, August-October 2019. MMWR Morb Mortal Wkly Rep 2019;68(45):1040-1041.

20. Crotty Alexander LE, et al. NIH workshop report: E-cigarette or vaping product use associated lung injury (EVALI): developing a research agenda [published online April 3, 2020]. Am J Respir Crit Care Med. https://doi.org/10.1164/ rccm.201912-2332ws. 